\title{
Correlation of the Physicochemical Properties in Soils from Tenerife Island, Measuring the Electric Current in an Electrokinetic Experiment ${ }^{\dagger}$
}

\author{
M.V. Vázquez, ${ }^{1}$ C.D. Arbelo, ${ }^{2}$ F. Hernández-Luis, ${ }^{3,}{ }^{*}$ D. Grandoso, ${ }^{3}$ M. Lemus ${ }^{3}$ \\ ${ }^{1}$ Grupo de Electroquímica, Instituto de Química, Facultad de Ciencias Exactas y Naturales, \\ Universidad de Antioquia, Medellín, Colombia \\ ${ }^{2}$ Departamento de Edafología y Geología, Universidad de La Laguna, Tenerife, España \\ ${ }^{3}$ Departamento de Química Física, Universidad de La Laguna, Tenerife, España
}

Received 25 April 2008; accepted 26 November 2008

\begin{abstract}
In this work the physical-chemical properties of six different soils original from Tenerife Island, Spain, are related with the measured electrical current in an electrokinetic experiment. Results show that total electrical charge has relation with properties such as: total metal content, $\mathrm{pH}$, carbon organic content, and cationic exchange capacity. Since all these properties are between those which allow to evaluate the fertility of a soil, an electrokinetic experiment with samples of this soil is proposed like diagnosis tool.
\end{abstract}

Keywords: electrokinetics, soil, electrolysis current, cationic exchange capacity.

\section{Introducción}

Es bien sabido que al aplicar técnicas electrocinéticas para la eliminación de contaminantes presentes en un suelo, proceso conocido como electrorremediación, resulta necesario analizar la evolución con el tiempo de algunas magnitudes fisicoquímicas y eléctricas relacionadas con el proceso, con el fin de mejorar su rendimiento.

Entre estas magnitudes, la corriente de electrólisis, cuando se trabaja a potencial aplicado constante, es una de las reportadas habitualmente en la literatura científica [1-12].

Entre otros, podemos destacar estudios encaminados a la eliminación de metales pesados tales como $\mathrm{Pb}, \mathrm{Cd}, \mathrm{Cr}, \mathrm{Ni}, \mathrm{Cu}$ y $\mathrm{Zn}$ [1, 5, 7, 8-12], en los que la corriente

\footnotetext{
† Dedicado a la Dra. Gloria Pastor Pinto y al Dr. José Acosta Rubio por su jubilación.

* Corresponding author. E-mail address: ffhelu@ull.es
} 
se relaciona con el rendimiento de la eliminación. Por otra parte, estudios sobre la disminución de la salinidad de suelos o la utilización de agentes complejantes como EDTA [2-3] para favorecer la eliminación de metales, han recurrido al análisis de la corriente de electrólisis con respecto al tiempo, para discutir sus resultados. También se ha utilizado dicho análisis en experimentos de electrorremediación que utilizan membranas selectivas a cationes en el proceso de eliminación de contaminantes [4], en estudios de la influencia de la capacidad buffer del suelo sobre el rendimiento de la eliminación [10] o en el estudio de procesos de eliminación múltiple simultánea [7].

Por último, cabe decir que en los últimos años, nuestro grupo de investigación ha llevado a cabo una serie de experiencias de "electro-caracterización" de suelos [13-18], en los cuales también se hace uso de la medida de la corriente de electrólisis. El procedimiento consiste en la aplicación de un campo eléctrico constante a muestras de diferentes tipos de suelos, registrando la corriente que circula por la célula y relacionándola con distintas propiedades del suelo, como pueden ser el contenido total de metales mayoritarios, $\mathrm{pH}$, conductividad eléctrica, contenido de carbono orgánico, capacidad de intercambio catiónico, granulometría, etc.

En suelos naturales (sin contaminar), la corriente de electrólisis varía con el tiempo de tratamiento electrocinético y presenta un perfil característico del mismo. Este perfil puede asociarse, entre otros factores, a la presencia de iones de distinta movilidad (distinto grado de retención por las partículas del suelo) o al comportamiento del suelo ante la presencia de los frentes ácido y alcalino electro-generados en las cámaras de los electrodos, como consecuencia del proceso de oxidación y reducción del agua.

La presencia de cationes móviles en el suelo, especialmente los denominados mayoritarios $\left(\mathrm{Na}^{+}, \mathrm{K}^{+}, \mathrm{Ca}^{2+}\right.$ y $\left.\mathrm{Mg}^{2+}\right)$, determinan la magnitud de algunos parámetros rutinariamente utilizados para caracterizar suelos de interés agrícola, tales como la capacidad de intercambio catiónico, grado de salinidad, conductividad eléctrica, etc.

La posibilidad de obtener este tipo de información mediante técnicas electrocinéticas, puede resultar de especial interés desde el punto de vista agrícola, dado que se trata de un método dinámico, que podría estar más cerca de los procesos que realmente ocurren en la naturaleza, que un método estático basado en otro tipo de análisis. Se trata pues de aproximarnos lo más posible, a una descripción del suelo que represente lo que ocurre en el mismo, incluyendo los equilibrios químicos simultáneos que se llevan a cabo en su seno.

En este trabajo se describe, brevemente, el procedimiento de "electrocaracterización" y se presentan algunos resultados preliminares obtenidos con seis muestras de suelos de la isla de Tenerife (España). Para cada uno de ellos, se analizó la variación de la corriente de electrólisis y se calculó la carga total que circuló. Dicha carga se correlacionó cualitativamente con algunas características del suelo. 
Tabla 1. Características de los suelos estudiados.

\begin{tabular}{|c|c|c|c|c|c|c|}
\hline & Ravelo & Junquito & Pachona & Los lirios & La laja & Erques \\
\hline \multirow{2}{*}{ Clasificación (USDA, 1999) } & Ultic & Rhodic & Leptic & Typic & Alfic & Lithic \\
\hline & Fulvudands & Paleustalfs & Calcitorrerts & Vitritorrands & Haplustans & Torriorthnts \\
\hline $\mathrm{pH}\left(\mathrm{H}_{2} \mathrm{O}\right)$ & 5,6 & 6,5 & 7,8 & 8,5 & 5,7 & 7,2 \\
\hline $\mathrm{C}\left(\mathrm{g} \mathrm{kg}^{-1}\right)$ & 90,4 & 24,0 & 15,0 & 18,2 & 20,9 & 25,0 \\
\hline $\mathrm{Ca} \quad\left(\mathrm{cmol}_{\mathrm{c}} \mathrm{kg}^{-1}\right)$ & 5,4 & 6,0 & 12,7 & 35,2 & 9,2 & 11,1 \\
\hline $\operatorname{Mg}\left(\mathrm{cmol}_{\mathrm{c}} \mathrm{kg}^{-1}\right)$ & 2,8 & 3,4 & 6,8 & 5,9 & 4,4 & 6,1 \\
\hline $\mathrm{Na} \quad\left(\mathrm{cmol}_{\mathrm{c}} \mathrm{kg}^{-1}\right)$ & 0,5 & 2,5 & 1,4 & 19,3 & 0,9 & 1,2 \\
\hline $\mathrm{K} \quad\left(\mathrm{cmol}_{\mathrm{c}} \mathrm{kg}^{-1}\right)$ & 0,9 & 1,5 & 2,2 & 22,5 & 1,7 & 2,6 \\
\hline $\operatorname{CEC~}\left(\mathrm{cmol}_{\mathrm{c}} \mathrm{kg}^{-1}\right)$ & 53,9 & 32,0 & 44,4 & 53,4 & 23,7 & 26,7 \\
\hline \multicolumn{7}{|l|}{ Análisis granulométrico } \\
\hline$\%$ Arena & 6,5 & 4,7 & 32,9 & 62,1 & 36,4 & 53,1 \\
\hline$\%$ Limo & 73,0 & 20,7 & 35,2 & 23,5 & 26,5 & 33,1 \\
\hline$\%$ Arcillas & 19,6 & 74,6 & 31,9 & 14,4 & 37,0 & 13,8 \\
\hline
\end{tabular}




\section{Materiales y Métodos}

\section{Suelos}

El estudio fue realizado con diversos suelos de origen volcánico de la Isla de Tenerife (España). En la Tabla 1 se presentan algunas características de los mismos. Previo a los experimentos electrocinéticos, unos $200 \mathrm{~g}$ de suelo fueron secados a temperatura ambiente $\left(25^{\circ} \mathrm{C}\right)$ durante 4 días y tamizados a tamaño de partícula de $2 \mathrm{~mm}$.

\section{Celda de electro-caracterización}

La celda utilizada es similar a las empleadas en trabajos previos [13-18]. Su esquema se muestra en la Figura 1, siendo las dimensiones del cilindro que contiene la pasta suelo/agua: $20 \mathrm{~cm}$ de largo por $2,5 \mathrm{~cm}$ de diámetro. Los electrodos utilizados fueron barras de grafito de $8 \mathrm{~cm}^{2}$ de área expuesta a la disolución.

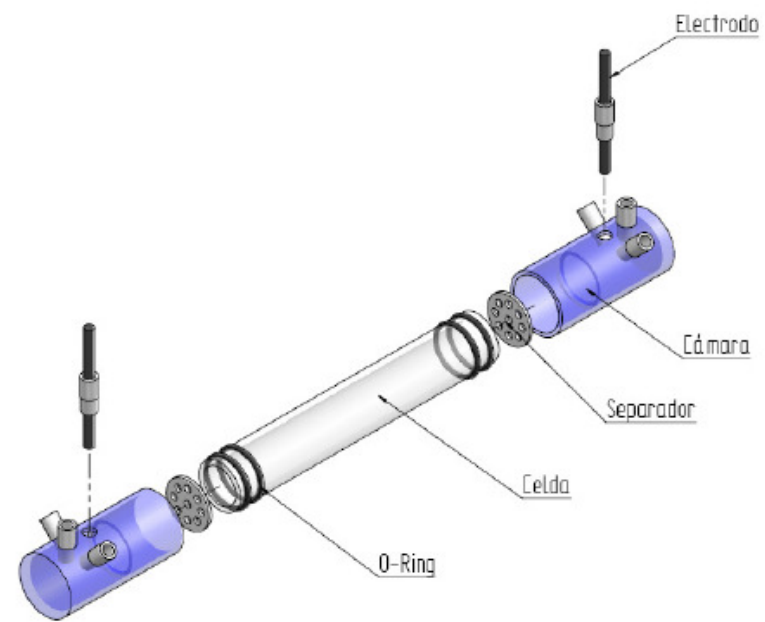

Figura 1. Esquema de la celda empleada para el estudio.

\section{Preparación de las muestras y tratamiento electrocinético}

Las muestras de suelo se dispusieron siguiendo el procedimiento utilizado en trabajos previos y que, básicamente, consiste en la preparación de una pasta húmeda con agua desionizada, que se deja en reposo durante 24 horas y luego se introduce en las celdas para el posterior tratamiento electrocinético. La relación suelo/agua se elige empíricamente de tal manera que las pastas tengan una consistencia semi-sólida adecuada para el empaquetamiento en las células (1,2 a $1,7 \mathrm{~g} \mathrm{~cm}^{-3}$ de densidad, dependiendo del tipo de suelo). El tratamiento electrocinético se realiza aplicando entre los electrodos de grafito, un campo eléctrico constante de $0,6 \mathrm{~V} \mathrm{~cm}^{-1}$ durante 48 horas, tiempo durante el cual se registra la corriente de electrólisis.

\section{Resultados y discusión}

La Fig. 2 muestra la variación de la corriente de electrólisis con el tiempo, para los seis suelos estudiados en este trabajo. Los resultados representados corresponden a un valor promedio de, al menos, tres experimentos. Como puede 
verse, en todos los casos se produce un fuerte incremento de la corriente en las primeras horas del experimento, para luego alcanzar un valor casi estacionario característico del tipo de suelo estudiado. Asumiendo que el sistema cumple la ley de Ohm, puede obtenerse un perfil similar de variación de la resistencia con el tiempo. En ese caso, se observa una disminución de la resistencia del medio en la primera hora para luego alcanzar un valor estacionario.

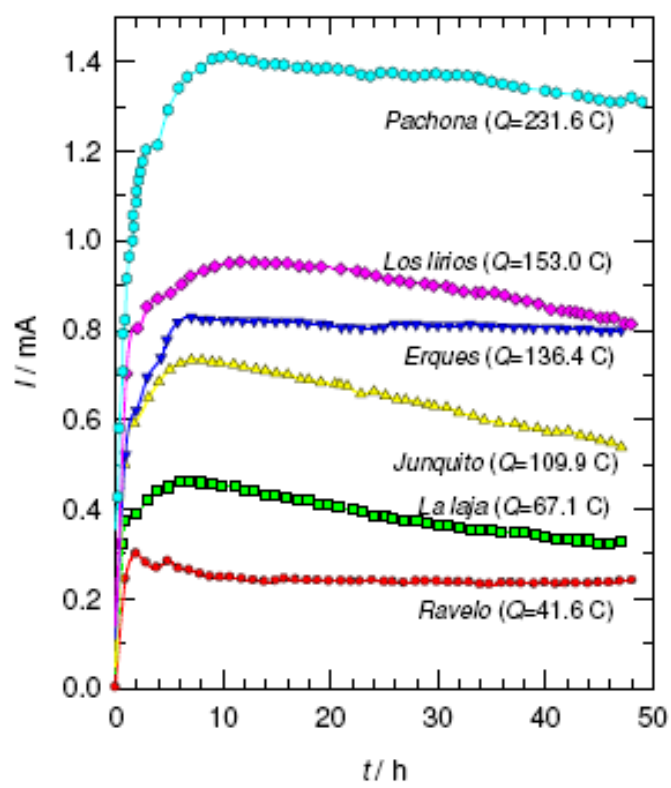

Figura 2. Variación de la corriente de electrólisis con el tiempo de tratamiento.

Este perfil de variación se debe a que, pasado un cierto tiempo (10-15 horas), es el segmento intermedio de la célula, que contiene la pasta suelo/agua, el que controla la velocidad del proceso. Como la conductividad eléctrica de cada suelo es distinta, también lo son los perfiles de variación, tanto de la corriente como de la resistencia.

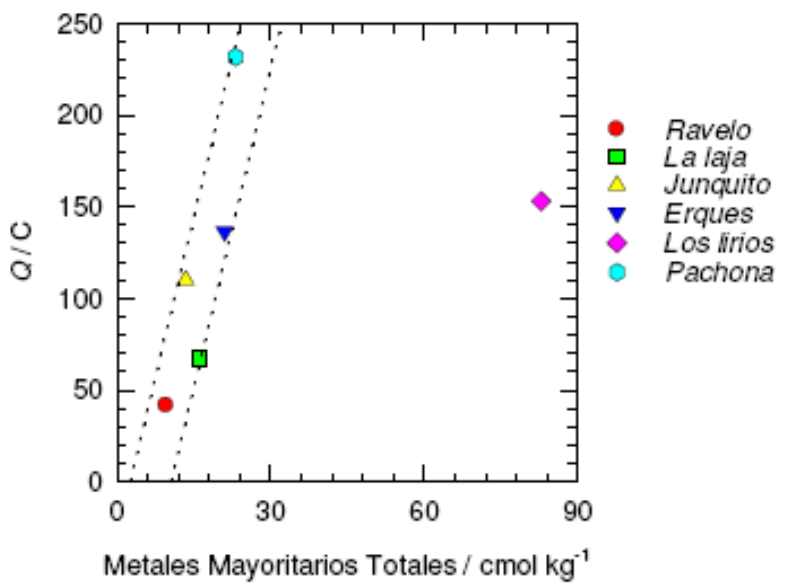

Figura 3. Relación entre la carga de electrólisis y el contenido de cationes mayoritarios $\left(\mathrm{Na}^{+}, \mathrm{K}^{+}, \mathrm{Ca}^{2+} \mathrm{y} \mathrm{Mg}^{2+}\right)$ en los suelos estudiados. 


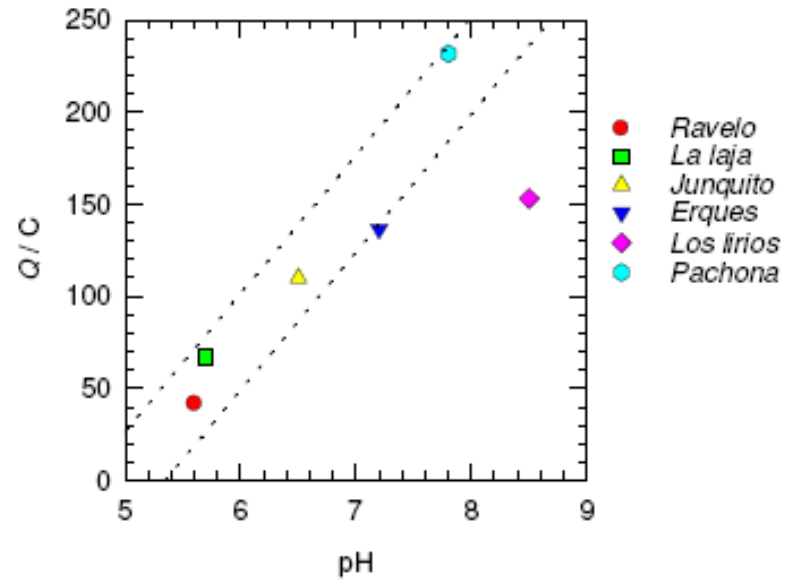

Figura 4. Relación entre la carga de electrólisis y el pH de los suelos bajo estudio.

En la Fig. 2 se muestra también, para cada uno de los suelos, la carga (área bajo la curva) que ha circulado por la célula en las 48 horas que duró el proceso. Este parámetro será el que intentaremos correlacionar cualitativamente con las características propias de cada suelo y, en especial, con la presencia de iones en el mismo.

Así, en la Fig. 3 se muestra la variación de la carga con el contenido de cationes mayoritarios $\left(\mathrm{Na}^{+}, \mathrm{K}^{+}, \mathrm{Ca}^{2+}\right.$ y $\left.\mathrm{Mg}^{2+}\right)$ presentes en la mezcla suelo/agua. La tendencia observada indica que la presencia de estos iones mayoritarios tiene una influencia importante en el valor de carga, salvo en el suelo denominado Los lirios. Este mismo suelo, que es el que mayor $\mathrm{pH}$ presenta de los estudios llevados a cabo, también se aleja de la tendencia mostrada en la Fig. 4, para la variación de la carga que ha circulado, con el pH del medio estudiado. Este anómalo comportamiento puede ser atribuido a la notable presencia de carbonatos en este suelo. Así, la tendencia mostrada en la Fig. 3 sería válida para suelos que no tengan mucho contenido de caliza.

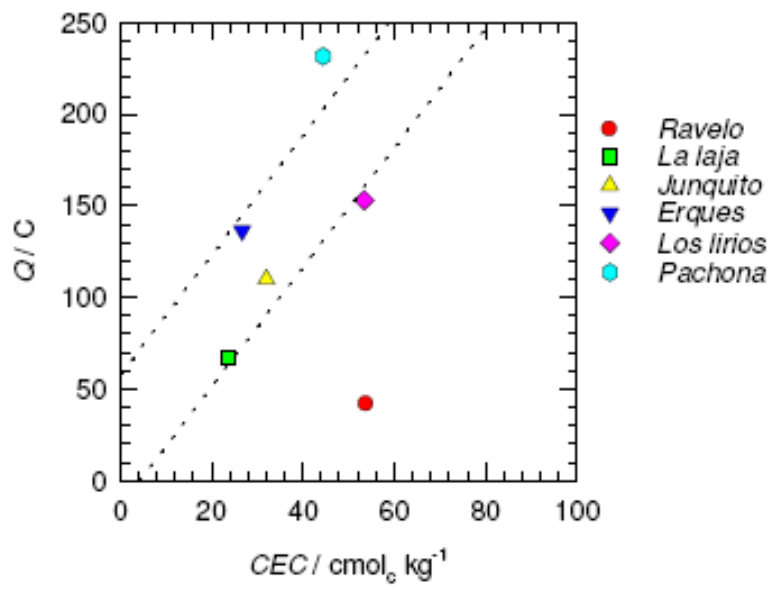

Figura 5. Relación entre la carga de electrólisis y la capacidad de intercambio catiónico de los suelos bajo estudio.

En la Fig. 5 se muestra la variación de la carga con la capacidad de intercambio catiónico del suelo. Dentro de la dispersión mostrada por los puntos, podría considerarse que existe una proporcionalidad entre ambos parámetros, excepto 
para el suelo denominado Ravelo. Este suelo presenta una cantidad de materia orgánica que excede en mucho los valores correspondientes a los otros suelos estudiados y se aleja notablemente del perfil de variación de la carga con la cantidad de materia orgánica medida como carbono total, como se muestra en la Fig. 6. Esto se explicaría teniendo en cuenta que la materia orgánica presente en el medio, ejerce un decisivo control sobre los equilibrios de intercambio catiónico dado que, por su capacidad complejante, impedirá que parte de los iones presentes puedan ser movilizados libremente bajo la acción del campo eléctrico.

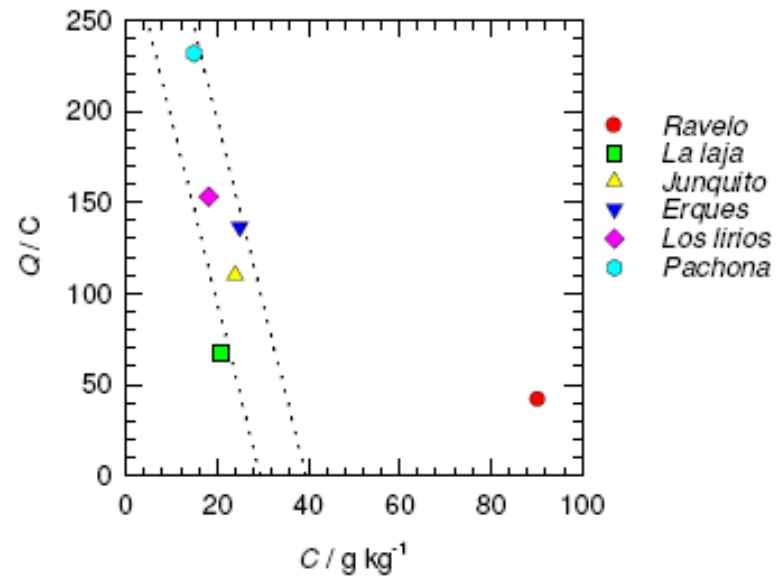

Figura 6. Relación entre la carga de electrólisis y el contenido de carbono orgánico en los suelos bajo estudio.

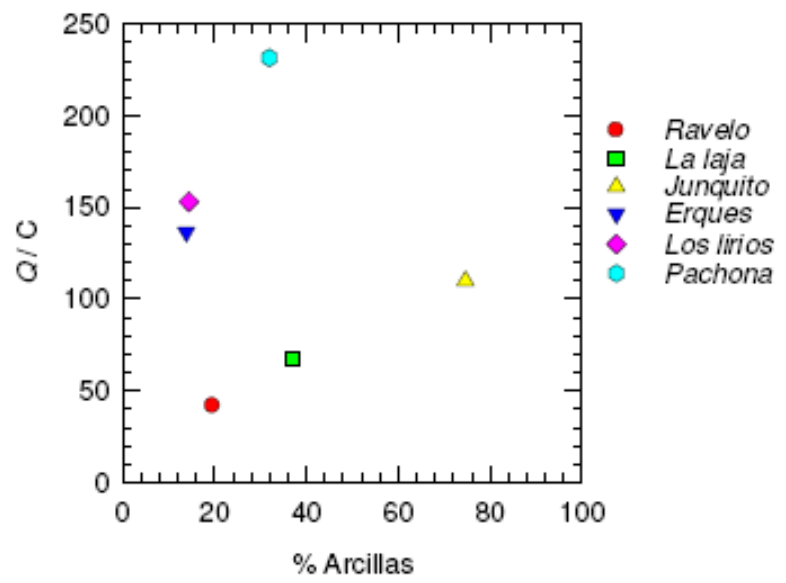

Figura 7. Variación de la carga de electrólisis con el contenido de arcillas de los suelos estudiados.

Por último en la Fig. 7 mostramos la dependencia de la carga transportada con el $\%$ de arcilla contenido en el suelo. Aunque la correlación parece casi imposible, se observan dos grupos de variación bien diferenciados para los que si parece haber una tendencia casi lineal. Por un lado, los suelos con pH ácido (Ravelo, Junquito y La Laja) y por otro, aquellos con pH básico (Pachona, Los lirios y Erques). La explicación de este hecho no resulta obvia y requiere un estudio con mayor profundidad. 


\section{Conclusiones}

Analizando las distintas relaciones mostradas en este trabajo, se puede considerar que, en principio, es factible utilizar la carga de electrólisis como criterio de caracterización de suelos.

Estos primeros resultados, preliminares, obtenidos al analizar la variación de la corriente de electrólisis con las distintas propiedades de los suelos bajo estudio, permiten afirmar que este parámetro electrocinético de fácil medida y obtenido en condiciones dinámicas, podría ser utilizado como herramienta de caracterización y diagnóstico para suelos de cultivo.

No obstante, deben hacerse muchos más estudios con el fin de poder racionalizar estas dependencias.

\section{Agradecimientos}

Los autores desean expresar su agradecimiento a la ORI (Oficina de Relaciones Internacionales) de la Universidad de La Laguna, por el apoyo brindado para la permanencia de investigadores de la Universidad de Antioquia en España, así como al Vicerrectorado de Investigación y Desarrollo Tecnológico, por la ayuda prestada a través de su Convocatoria de Ayudas para el Mantenimiento de Grupos de Investigación Consolidados ( $\mathrm{N}^{\mathrm{o}}$ 1802440702).

Se agradece también a la Universidad de Antioquia, a través del CODI, el apoyo dado para el desarrollo de este trabajo.

\section{Correlación de propiedades fisicoquímicas de suelos de la Isla de Tenerife, con la corriente eléctrica medida en un experimento electrocinético}

\section{(Dedicado a la Dra. Gloria Pastor Pinto y al Dr. José Acosta Rubio por su jubilación)}

\section{Resumen}

En este trabajo se relacionan las propiedades fisicoquímicas de seis suelos diferentes, originarios de la Isla de Tenerife, España, con la corriente eléctrica medida en un experimento electrocinético. Los resultados muestran que la carga eléctrica total tiene relación con propiedades tales como: contenido total de metales mayoritarios, $\mathrm{pH}$, contenido de carbono orgánico y capacidad de intercambio catiónico. Dado que todas estas propiedades están entre las que permiten evaluar la fertilidad de un suelo, se propone un experimento electrocinético con muestras de dicho suelo como herramienta de diagnóstico.

Palabras clave: electrocinética, suelo, corriente de electrólisis, capacidad de intercambio catiónico.

\section{Referencias}

1. Z. Li, J-W. Yu, and I. Neretnieks, J. Hazard. Mater. 55 (1997) 295.

2. S. Pamukcu, A. Weeks, and J.J. Wittle, J. Hazard. Mater. 55 (1997) 305.

3. J.S.H. Wong, R.E. Hicks and R. Probstein, J. Hazard. Mater. 55 (1997) 61.

4. Z. Li, J-W. Yu, and I. Neretnieks, Environ. Sci. Technol. 32 (1998) 394. 
5. $\quad$ K.R. Reddy and S. Chinthamreddy, Waste Manage. 19 (1999) 269.

6. S. Chinthamreddy and K.R. Reddy, J. Soil Contam. 8 (1999) 197.

7. K.R. Reddy, S. Chinthamreddy and A. Al-Hamdan, Remediation 11 (2001) 85.

8. $\quad$ S.I. Darmawan and S.I. Wada, Appl. Clay Sci. 20 (2002) 283.

9. V. Pomes, A. Fernández and D. Houi, Process Saf. Envirom. 80 (2002) 256.

10. K.R. Reddy and A. Chinthamreddy, J. Geotech. Geoenviron. 129 (2003) 263.

11. D-M. Zhou, C-F. Deng and C. Long, Chemosphere 56 (2004) 265.

12. M. Pazos, A.A. Sanromán and C. Cameselle, Chemosphere 62 (2006) 817.

13. F. Hernández-Luis, M. Lemus, D. Grandoso, M.V. Vázquez and C.D. Arbelo, Remediation 16 (2005) 107.

14. F. Hernández-Luis, D.M. Benjumea and M.V. Vázquez, Innovación 16 (2004) 7.

15. F. Hernández-Luis, M. Lemus, D. Grandoso, M.V. Vázquez and C.D. Arbelo, Remediation 14 (2004) 105.

16. M.V. Vázquez, F. Hernández-Luis, D. Grandoso, and C.D. Arbelo, Port. Electrochim. Acta, 22 (2004) 387.

17. M.V. Vázquez, F. Hernández-Luis, M. Lemus and C.D. Arbelo, Port. Electrochim. Acta 22 (2004) 399.

18. M.V. Vázquez, F. Hernández-Luis, D. Benjumea, D. Grandoso, M. Lemus and C.D. Arbelo, Sci. Total Environ. 378 (2007) 214. 